Pediat. Res. 4: 286-294 (1970)

Electromyography

hyperkalemic periodic paralysis

muscle myotonia

potassium

water, intracellular

\title{
Arterial-venous Differences in Potassium Concentrations in Serum in Paramyotonia
}

\author{
R. KLEIN ${ }^{[17]}$ and J.E. HADDOW \\ Department of Pediatrics, Boston University School of Medicine and Boston City Hospital, \\ Boston, Massachusetts, USA
}

\section{Extract}

A group of patients is described with paramyotonia congenita whose condition was marked by a negative arterial-venous (A-V) difference in potassium concentration in plasma under basal conditions. The means \pm SEM for arteriolized capillary blood, venous blood, and the differences between them were $4.13 \pm 0.13,5.05 \pm 0.10$, and $-0.48 \pm 0.08 \mathrm{mEq} /$ liter, respectively. The $\mathrm{A}-\mathrm{V}$ potassium difference in plasma becomes positive during exacerbation of signs and symptoms. The corresponding means \pm SEM were $4.74 \pm 0.17,4.66 \pm 0.15$, and $+0.08 \pm 0.03 \mathrm{mEq} /$ liter. The symptoms are precipitable by either chilling or administering potassium. Attacks of either myotonia or paresis were not associated with spontaneous hyperkalemia. The muscles of these patients contain increased amounts of water and sodium and decreased amounts of potassium.

\section{Speculation}

It is believed that the negative A-V potassium difference in plasma constitutes a marker that enables patients with paramyotonia congenita to be separated from patients with hyperkalemic periodic paralysis and myotonia. In the latter instance, myotonia is also precipitable by chilling and relieved by rest. There is a correlation between increased muscle water between attacks and the occurrence of myotonia in patients with the various forms of periodic paralysis.

Introduction

Individuals from two families and two unrelated patients with paramyotonia congenita whose condition is marked by a greater concentration of potassium $(\mathrm{K})$ in venous serum or plasma than in arteriolized capillary serum or plasma when the patients are asymptomatic was studied. It is hoped that measurement of negative arterial-venous (A-V) difference in serum potassium may help clarify the nosology of the periodic paralyses. The related patients were members of two families previously thought to have hyperkalemic periodic paralysis.
Drager and co-workers [2] and van Der Meulen et al. [10] among others, have suggested that paramyotonia, hyperkalemic periodic paralysis with myotonia, and hyperkalemic periodic paralysis without myotonia form a continuum in a single disease. To these disease forms might be added sodium-responsive periodic paralysis (a condition not associated with myotonia) as the other instance of a potassium-inducible paralytic syndrome [8]. It seems valid to consider each as a separate entity with some common factor accounting for myotonia.

The paralysis in these syndromes is episodic and flaccid, occurring when the patient is at rest, and is 
inducible by potassium. Myotonia in these syndromes, in contrast to that of myotonia congenita, is produced or aggravated by exercise and relieved by rest. The unusual cases of periodic paralysis reported by LAYZER et al. [7] had myotonia like that present in myotonia congenita which wore off with activity. When myotonia is present, therefore, it is difficult to distinguish between these syndromes on clinical grounds.

In general, myotonia is more prominent in paramyotonia congenita, whereas paralysis predominates in hyperkalemic periodic paralysis. Paralysis is more readily produced in hyperkalemic periodic paralysis with or without myotonia by short periods of exercise followed by rest than in either paramyotonia or sodiumresponsive periodic paralysis $[4,8]$. These factors, however, are subjectively determined and are, therefore, not very helpful in differential diagnosis. Although paramyotonia was originally characterized by the precipitation of the myotonia by cold, in reality nearly all myotonia is precipitated or aggravated by cold. ToMPkINS et al. [9] have even produced myotonia in a healthy individual by cold immersion of a limb. We have produced repetitive intracellular action potentials in intact rats by external cooling of a limb [6]. As cooling progressed, repetitive action potentials disappeared and, eventually, the muscles became unresponsive to stimulation.

The distinguishing feature of hyperkalemic periodic paralysis noted in the laboratory is the spontaneous increase in $\mathrm{K}$ concentration in serum at the onset of paralysis. The A-V potassium difference in serum is often negative at this time. The rising $\mathrm{K}$ concentration in serum may not exceed the normal range, but frequently will elevate to between 5.5 and $7.5 \mathrm{mEq} / \mathrm{liter}$ [4]. Arterial-venous differences in $\mathrm{K}$ concentration have not previously been measured in either paramyotonia or the sodium-responsive syndrome, but in neither of these conditions have spontaneously elevated nor rising concentrations of $\mathrm{K}$ been reported. Negative evidence of this sort is not sufficient to establish that this observation distinguishes paramyotonia or the sodium-responsive syndrome from hyperkalemic periodic paralysis; however, it is felt that the evidence to be presented is sufficient to establish a distinction between paramyotonia congenita and hyperkalemic periodic paralysis with myotonia, albeit with the proviso that these findings be verified in other patients with paramyotonia congenita.

\section{Materials and Methods}

Subjects

Patient $A$, a 60-year-old man, first developed severe stiffness in his extremities at the age of 9 years while sledding. Flaccid weakness in his legs subsequently appeared, forcing him to remain in bed for 1 week, although he remained able to walk short distances. Several similar attacks occurred between the ages of 9 and 17 years. All began when he was sledding, although such conditions did not always produce symptoms. Between the ages of 35 and 60 years he had five episodes of flaccid paralysis. Three were spontaneous, occurring in the morning and associated with stiffness. One occurred on awakening from anesthesia for a stapedectomy. Myotonia was noted to be absent on this occasion by his physicians. His serum $\mathrm{K}$ level was $4.4 \mathrm{mEq} / \mathrm{liter}$ at this time. Concentration of $\mathrm{K}$ in serum at the height of a spontaneous attack was $4.3 \mathrm{mEq} / \mathrm{liter}$. The fifth episode was induced by intravenous administration of $53 \mathrm{mEq}$ of $\mathrm{K}$ which resulted in a $1.9 \mathrm{mEq} /$ liter increase in venous $\mathrm{K}$ levels to 6.3 $\mathrm{mEq} /$ liter. Myotonia was present at the start of this study but disappeared as he developed generalized flaccid paralysis lasting $4 \mathrm{~h}$. On two later occasions he reported unwitnessed periods of unconsciousness lasting several hours. These were not preceded by an aura or accompanied by incontinence but were associated with residual weakness for 2 days.

Physical examination showed him to be well developed, with no baldness, testicular atrophy, or muscle hypertrophy. He did have mild thigh muscle wasting and difficulty in climbing stairs. Lid lag and slight ptosis of the eyelids were present. There was nearly constant percussion myotonia of the tongue and pectorals and percussion myotonia was intermittently noted in all muscles tested. He had no difficulty in relaxing his grasp. There was no documentation of similar disease in his family.

Patient B-1, a 13-year-old boy, complained of frequent transient stiffness after exercise in cold weather. This occurred between the ages of 4 and 9 years. Again, exposure to cold did not invariably produce symptoms. From the age of 9 years, he began having increasingly frequent exercise-induced attacks even in warm weather, forcing him to give up participation in competitive sports. His physical appearance was unremarkable and his musculature was normal in appearance. Lid lag was constantly demonstrable and percussion myotonia was usually demonstrable at rest. He had no trouble relaxing his grasp. His tolerance to climbing stairs varied from two to eight flights after which his legs stiffened and his quadriceps became strikingly hard. Percussion myotonia was easily demonstrable in his lower extremities at such times but often could not be simultaneously elicited in his arms.

Patient B-2, the 20-year-old brother of patient $B-1$, was normally developed and muscled. His myotonia gradually improved during adolescence with no residual limitation in his activities. Percussion myotonia was 
still frequently present, however, although he had had only one severe myotonic attack over the past 3 years, two of which he spent on active duty in the National Guard. He allegedly had had attacks of flaccid paresis of his legs in the past, which he noted as a buckling of the knees and inability to stand, lasting for less than $1 \mathrm{~h}$.

Patient B-3, a 17-year-old sister of patient B-2, had myotonia only on swimming in very cold water or when otherwise severely chilled. She stated that attacks could be aborted rapidly by ingesting carbohydrates. Her physical examination was unremarkable.

Four other siblings and the mother in the B family were reportedly unaffected. The father began to have myotonic symptoms at age 21 . He was not significantly inconvenienced by his condition.

Patient $C$ was 54 years old and was a well-developed, muscular Negro male. Four years previously he had had a spinal fusion for a ruptured intervertebral disc. Because of residual numbness in one leg, the onset of myotonia could not be timed with certainty. Incapacitating stiffness, particularly in the affected leg, had bcgun sometime in the last 2 years. Percussion myotonia was readily demonstrated in nearly all muscles. $\mathrm{He}$ had no lid lag, and there was no known family history of muscular disease. His children were not examined.

Patient $D$ was a 10 -year-old boy whose family history has been reported [10]. He had percussion myotonia without other findings on physical examination. $\mathrm{He}$ was not inconvenienced by his condition.

The methods used in these studies have been previously reported $[5,6]$. Sodium and potassium were determined with a flame photometer using an internal lithium standard, chloride with a Cotlove chloridometer, and calcium and magnesium with an atomic absorption spectrophotometer. Enzyme activities in excised muscle (patient $A$ ) were measured by Dr. Davio Dawson [15]. Measurements of aldosterone in urine were made by a commerical laboratory [12]. Blood specimens were obtained from an antecubital vein and from a warmed finger tip on the same side [13]. The attempt was made to obtain the specimens simultaneously. When there was more than $1 \mathrm{~min}$ difference in time, the individual times for each specimen are reported with the results. Electrolyte concentrations in such 'arteriolized' capillary specimens have been found to be similar to arterial concentrations [5]. The so-called 'basal' values represent specimens obtained in the morning, in the fasting and the postprandial states, or in two instances, in the early afternoon $1 \mathrm{~h}$ after lunch. At either time, preceding exercise had been limited to a short walk. Patients were well rested and asymptomatic, and the signs of myotonia were either absent or minimal for that patient. Patient $B-I$ always had a demonstrable lid lag and patient $C$ always had mild percussion myotonia. Control subjects were all tested in the morning; slightly more than one-half of the control subjects were in the fasting state; they had not exercised and were at rest. Cooling was produced in the muscles of the hand by swathing the hand in an ice bag or immersing it in iced water until the skin temperature was $19-21^{\circ}$.

\section{Results}

\section{Clinical Summary}

Myotonia, the predominant symptom in our patients, was aggravated by exercise and relieved by rest. Typical of this condition, it was precipitated or aggravated by exposure to cold. The sign, percussion myotonia, was almost always demonstrable. Symptomatic myotonia occurred independently of time of day. Most commonly, myotonic symptoms subsided with rest within $30 \mathrm{~min}$. The speed of resolution varied inversely with the severity of the attack. One patient reported that carbohydrate ingestion hastened relief from myotonia. No benefit was noted from increased salt intake by one patient or from administration of chlorothiazide to two other patients. No patient had food cravings or aversions.

In contrast to the myotonia, flaccid paralysis occurred infrequently but lasted as long as several days. It was likely to occur on awakening in the morning and was never produced by exposure to cold. None of the patients knew of any way to hasten recovery from paralysis.

Experimentally, the ingestion or intravenous infusion of potassium chloride precipitated myotonia and occasionally caused flaccid paralysis. This effect could not be predictably reproduced in a given patient. When paralysis did ensue, myotonia disappeared only to reappear as paralysis subsided. Symptoms could not be induced by exercise followed by rest. In the two kindreds with multiple affected members, the condition was inherited as an autosomal dominant trait with onset most commonly in the first two decades.

Electromyography. Typical decrescendo repetitive potentials (dive-bomber effect) were recorded on insertion of intramuscular electrodes in patients $A, B-1$, and $C$. Electromyography using skin electrodes revealed only polyphasic potentials following electrical and mechanical stimuli in patients $A, B-1, B-2$, and $B-3$. The most striking finding of surface electromyography in patient $C$ was the almost continuous activity that was recorded on two occasions. The activity would cease following an action potential and contraction produced by percutaneous electrical stimulation of the motor nerve. Oral administration of $200 \mathrm{~g}$ glucose also was 
associated with a return to an electrically silent state after approximately $20 \mathrm{~min}$.

When muscles that were being tested were swathed in an ice bag until skin temperature was $19-21^{\circ}$, prolongation in duration of the action potential was greater than that seen in normal individuals studied in a similar manner (fig. 1). In patients $B-2$ and $B-3$, cooling increased the number and duration of polyphasic action potentials.

Electrolyte concentrations in plasma under basal conditions. Tables I and II present data for capillary and venous concentrations of electrolytes, $\mathrm{H}_{2} \mathrm{O}$, hexose, and osmolality under basal conditions. Mean capillary $\mathrm{K}$ concentration in plasma in 18 healthy children and young adults was $4.72 \pm 0.28$ (SD) $\mathrm{mEq} /$ liter, mean venous $\mathrm{K}$ concentration was $4.59 \pm 0.35 \mathrm{mEq} / \mathrm{liter}$. The mean $\mathrm{A}-\mathrm{V}$ potassium difference in plasma in these normal subjects was $+0.1 \pm 0.17 \mathrm{mEq} /$ liter. In patients under the same conditions, the mean capillary $\mathrm{K}$ concentration in plasma was $4.53 \pm 0.40 \mathrm{mEq} / \mathrm{liter}$ and the corresponding mean venous concentration was $5.05 \pm 0.31 \mathrm{mEq} /$ liter. The mean $\mathrm{A}-\mathrm{V}$ difference was $-0.5 \pm 0.26 \mathrm{mEq} /$ liter. Each individual value differed from the mean normal value of $+0.1 \pm 0.17$ by more than two standard deviations (table I).

Factors affecting plasma electrolytes. No spontaneous elevations of $\mathrm{K}$ concentrations in serum have been demonstrated in these patients during symptomatic myotonia attacks or paralytic episodes either by ourselves or by other physicians.

Table III presents data obtained before and after exercise induced severe symptomatic myotonia in patient $B-1$ on two occasions. The exercise was climbing three and eight flights of stairs as rapidly as possible. Also presented are similar data obtained when patient $A$ was seen during an attack of flaccid paralysis. The $\mathrm{A}-\mathrm{V}$ potassium difference reverted to normal after strenuous exercise in patient $B-1$.

Flaccid paralysis in patient $A$ began 2 days after discontinuation of treatment with acetazolamide. He had generalized paresis, most marked on the left side. His left arm was almost completely flaccid. Deep tendon reflexes were markedly depressed on the left. Percussion myotonia was prominent in the right arm and pectorals but absent on the left. Capillary and venous $\mathrm{K}$ concentrations in plasma were both $4.8 \mathrm{mEq} / \mathrm{liter}$. $\mathrm{He}$ was given $100 \mathrm{mg}$ of the sodium salt of hydrocortisone hemisuccinate intravenously. Within $9 \mathrm{~min}$, there was a dramatic increase in strength of the left arm and a lesser increase of strength of the right arm. Within $20 \mathrm{~min}$, strength and deep tendon reflexes were approximately equal in both arms. At this point, the capillary $\mathrm{K}$ concentration was $4.7 \mathrm{mEq} /$ liter and the venous concentration was $5.7 \mathrm{mEq} /$ liter. Percussion myotonia continued in the right arm and became readily demonstrable in the left.

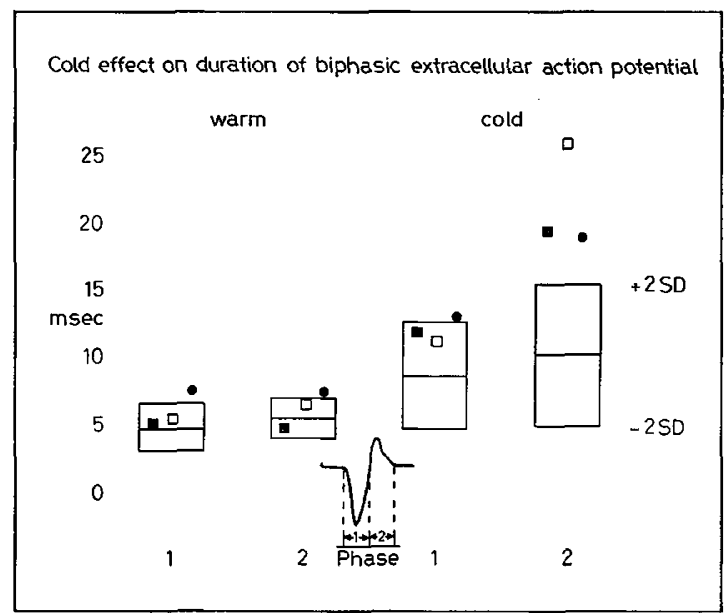

Fig. 1. Boxes represent means \pm SD for 13 normal young subjects for duration of first and second phases of biphasic extracellular action potentials before and after cooling abductor digiti quinti. Percutaneous stimulation of ulnar nerve at elbow was used. Delineation of the two phases is indicated by sketch in lower middle of figure. Cooling was by application of ice bag until skin temperature was $19-21^{\circ}$. The mean durations for at least three individual determinations in patients with periodic myotonia are represented by superimposed symbols. $\square$ : patient $B-1$. : : patient $D$. $\bullet$ : patient $A$. The duration of control action potentials for each patient is consistent with control subjects of the same age.

Table I. Arteriolized capillary and venous concentrations of potassium in plasma, and $\mathrm{A}-\mathrm{V}$ differences in control subjects and patients under basal conditions

\begin{tabular}{lccc}
\hline Subjects & \multicolumn{3}{c}{ Potassium, mEq/liter } \\
\cline { 2 - 4 } & Capillary & Venous & $\begin{array}{c}\text { Capillary- } \\
\text { venous } \\
\text { difference }\end{array}$ \\
\hline Controls (18) & & & \\
Patient $A$ & $4.7 \pm 0.28^{2}$ & $4.6 \pm 0.35^{2}$ & $0.1 \pm 0.17^{2}$ \\
& 4.5 & 5.0 & -0.5 \\
& 3.9 & 5.0 & -1.1 \\
& 4.4 & 4.7 & -0.3 \\
Patient $B-1$ & 4.8 & 5.4 & -0.6 \\
& 4.4 & 4.8 & -0.4 \\
Patient $C$ & 4.8 & 5.1 & -0.3 \\
& 4.0 & 4.6 & -0.6 \\
& 4.8 & 5.1 & -0.3 \\
& 5.0 & 5.5 & -0.5 \\
\hline
\end{tabular}

${ }_{1}$ Number in parenthesis indicates number of control subjects.

${ }^{2}$ Mean \pm SD. 





Arterial-venous potassium differences in paramyotonia
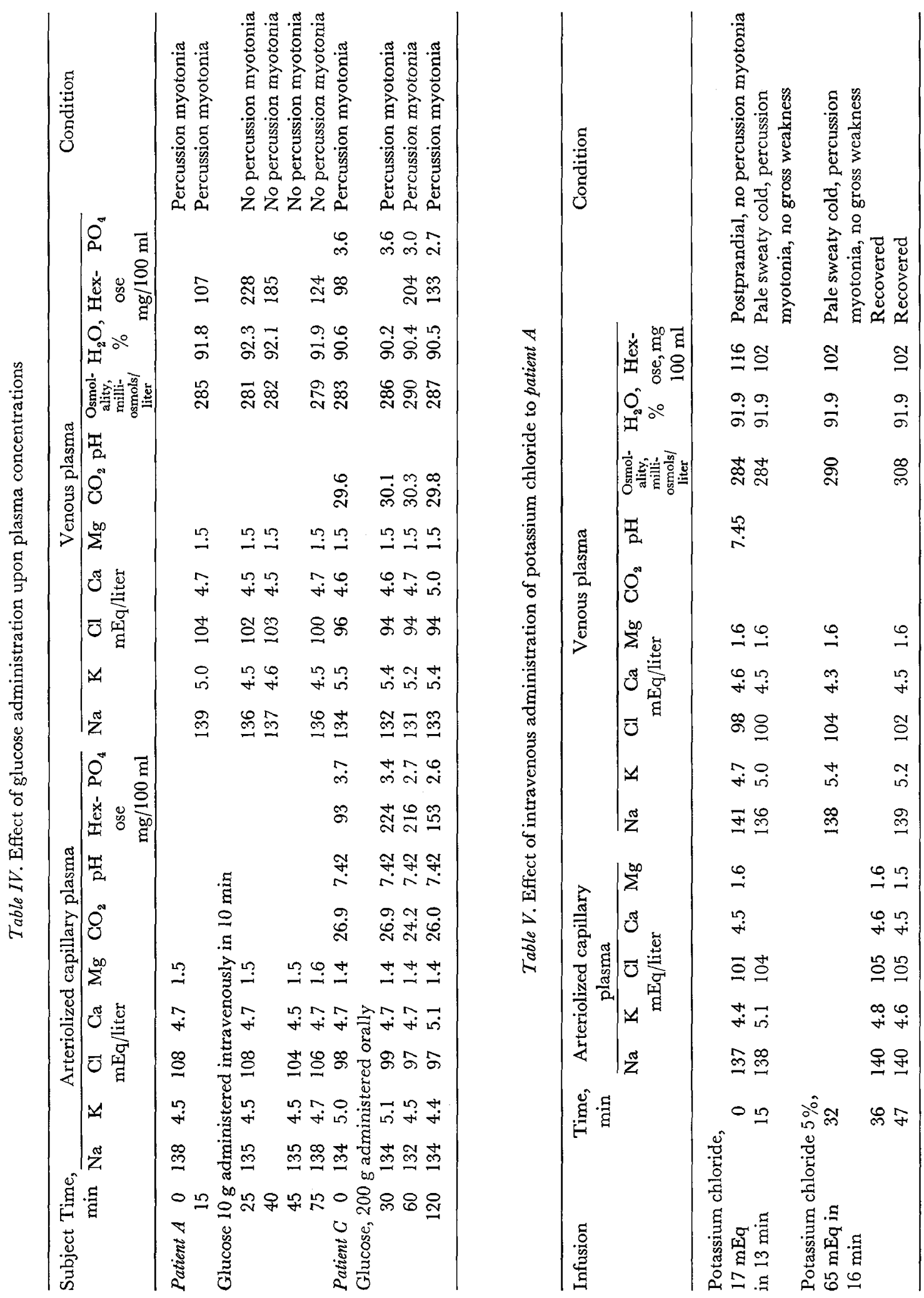
Figure 2 shows the effect in patient $A$, and also in a patient with familial hyperkalemic periodic paralysis with myotonia studied concurrently, of ambulation for $0.5 \mathrm{~h}$ followed by rest in a supine position for $0.5 \mathrm{~h}$. This procedure produced no observable change in patient $A$ but produced flaccid paralysis in the patient with hyperkalemic periodic paralysis. Indeed, at no time has it been possible to produce paresis or other symptoms in patients $A, B-1$, and $C$ by rest after exercise. It should be noted that venous $\mathrm{K}$ concentration in patient $A$ was consistently greater than capillary concentrations although he remained asymptomatic throughout the study. In contrast, the $\mathrm{A}-\mathrm{V}$ potassium difference became negative only in the patient with familial hyperkalemic periodic paralysis and myotonia at the onset of paralysis, coincident with the increase in plasma $\mathrm{K}$ concentrations. No significant change was noted in plasma concentrations of other electrolytes, osmolality, water, $\mathrm{pH}$, and hexose in either patient. Similar changes in the latter condition had been previously reported [5].

Table IV records the effects of intravenous and oral administration of glucose on plasma electrolytes. In patient $A$, intravenous infusion of glucose was followed by no change in capillary $\mathrm{K}$ concentration while the venous concentration decreased $0.5 \mathrm{mEq} / \mathrm{liter}$, returning the A-V difference to the normal positive state. Glucose ingestion by patient $C$ was associated with a greater negative $\mathrm{A}-\mathrm{V}$ difference in $\mathrm{K}$ concentration as the capillary value fell more than did the venous concentration in plasma.

When potassium chloride was infused intravenously in patient $A$, the $\mathrm{A}-\mathrm{V}$ potassium difference in plasma became positive when symptoms appeared, and reverted to negative as he improved with the infusion of hypertonic sodium chloride. The data are presented in table $\mathrm{V}$.

Muscle biopsy. A sample of muscle was obtained from patient $D$ and was analyzed within $30 \mathrm{~min}$ for water and electrolyte contents. A biopsy was also obtained under local anesthesia from the left deltoid of patient $A$. Care was taken to avoid infiltrating the muscle. At the time of the biopsies, both patients were asymptomatic except for percussion myotonia. Light and electronmicroscopic examination of the specimen from patient $A$ revealed no abnormalities. Enzyme activities of phosphorylase a (EG. 2.4.1.1), creatine kinase (EG. 2.7.3.2), lactate dehydrogenase (EC. 1.1.1.27), aldolase (EC. 4.1.2.7), and phosphoglucomutase (EG. 2.7.5.1) were normal. Anaerobic metabolism of glycogen, glucose 6-phosphate and fructose-di-phosphate was also normal as judged by lactate production.

Electrolyte and water values per kilogram of wet fat-free muscle for patients $A$ and $D$, as well as normal mean values and values from patients with hyperkale- mic periodic paralysis from our laboratory are recorded in table VI. Both groups had increased muscle water, sodium, and chloride and decreased potassium in muscle. The mean water and potassium values for muscle obtained from asymptomatic patients with paramyotonia congenita by DRAGER et al. [2] were $776 \mathrm{ml} \mathrm{H}_{2} \mathrm{O}$ and $88 \mathrm{mEq} \mathrm{K} / \mathrm{kg}$ wet fat-free muscle. It should be noted that although the three muscle samples from patients with hyperkalemic periodic paralysis studied by us contained diminished amounts of sodium and chloride, only one of four patients similarly studied by others did not have an increased amount of sodium and chloride in his muscle specimens [4].

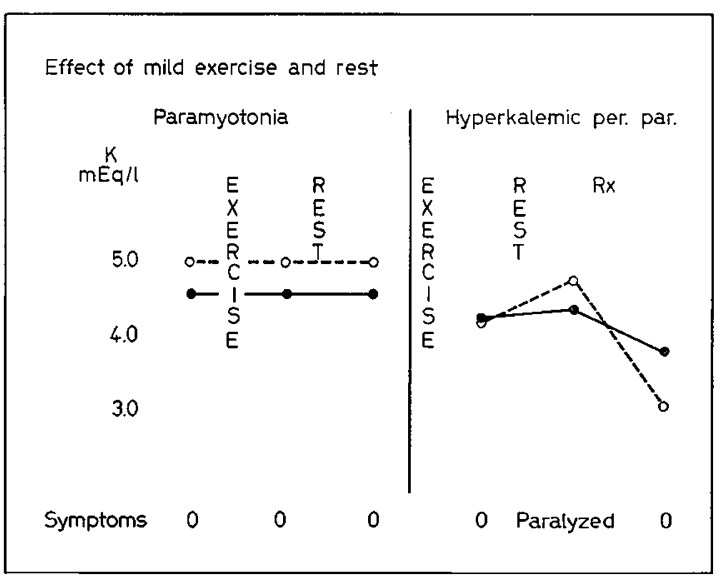

Fig. 2. - : capillary values. $\bigcirc---O$ : venous values. Exercise was $0.5 \mathrm{~h}$ ambulation on ward; rest was $0.5 \mathrm{~h}$ in a supine position; $\mathrm{Rx}$ was $0.4 \mathrm{ml} 1: 1,000$ epinephrine $\cdot \mathrm{HCl}$ administered subcutaneously.

Table VI. Electrolyte and water content of muscle in normal subjects and in patients with hyperkalemic periodic paralysis and paramyotonia

\begin{tabular}{|c|c|c|c|c|c|c|}
\hline \multirow[t]{2}{*}{ Subjects } & \multirow{2}{*}{$\begin{array}{c}\mathrm{H}_{2} \mathrm{O} \\
\mathrm{ml}\end{array}$} & $\mathrm{Na}$ & $\mathrm{K}$ & $\mathrm{Cl}$ & $\mathrm{Ca}$ & $\mathrm{Mg}$ \\
\hline & & \multicolumn{5}{|c|}{$\begin{array}{c}\mathrm{mEq} / \mathrm{kg} \text { wet fat-free } \\
\text { muscle }\end{array}$} \\
\hline Normals (12) ${ }^{1}$ & 758 & 35 & 95 & 21 & 4 & 17 \\
\hline $\begin{array}{l}\text { Hyperkalemia periodic } \\
\text { paralysis }(3)\end{array}$ & 773 & 27 & 91 & 18 & - & 一 \\
\hline \multicolumn{7}{|l|}{ Paramyotonia } \\
\hline Patient $A$ & 778 & 52 & 84 & 33 & 5 & 13 \\
\hline Patient $D$ & 788 & 41 & 81 & 33 & 2 & 14 \\
\hline
\end{tabular}

${ }^{1}$ Number in parentheses indicates number of patients. 
Miscellaneous. External cooling of muscle precipitated local myotonia in our patients with paramyotonia congenita or aggravated preexisting percussion myotonia. At times the same amounts of chilling had little effect. Excretion of aldosterone in urine on uncontrolled diets was normal in patients $A$ and $C$ as were glucose utilizations in the same patients. The EEG of patient $A$ was normal.

\section{Treatment}

Patient $B-1$ has been treated with $d$-amphetamine in prolonged action capsules for 1 year. The relation of arterial to venous $\mathrm{K}$ concentrations in serum became positive 1 week after initiation of therapy. Following treatment he was able to climb 18 flights of stairs and engage in vigorous exercise without symptoms. Percussion myotonia was no longer demonstrable, and lid lag was markedly reduced. On two occasions, administration of $d$-amphetamine was discontinued and symptoms returned within a day or two.

The A-V difference in potassium also became positive when patient $A$ was given $d$-amphetamine for 1 week. The side reactions to the drug were intolerable and there was no dramatic clinical improvement. Acetazolamide was administered for 9 months. Percussion myotonia was diminished, arterial and venous electrolyte concentrations in serum were normal, and there were no clinical attacks of myotonia. Strength in his legs, however, did not improve measurably. When acetazolamide was discontinued, he developed flaccid weakness after 2 days. Paresis was rapidly relieved by intravenous infusion of the sodium salt of hydrocortisone hemisuccinate as noted above.

\section{Discussion}

Clinically, these patients could be classified as having either paramyotonia congenita or hyperkalemic periodic paralysis with myotonia. Although the predominance of myotonia as a symptom and failure to produce weakness by ambulation followed by rest would tend to make us favor paramyotonia, these findings are not adequate to discriminate between the two diagnoses if they are separate entities. The values for muscle water and electrolytes when our patients were asymptomatic were similar to those reported for patients with paramyotonia by DRAGER et al. [2] and are unlike our findings in patients with hyperkalemic periodic paralysis. This argument suffers because of the variations in normal muscle water and electrolyte values reported by different laboratories, and because other investi- gators have not noted decreased sodium and chloride contents in muscle from patients with hyperkalemic periodic paralysis [4]. Although we are more certain of the validity of our own findings now that we have reproduced them 9 years later in a different laboratory, the explanation for the different results reported by other investigators remains moot. This leaves the A-V differences in $\mathrm{K}$ concentrations in plasma to separate paramyotonia from hyperkalemic periodic paralysis. We have not seen higher venous than capillary $\mathrm{K}$ concentrations in normal individuals or in patients with various forms of periodic paralyses when they were asymptomatic. ANDREs et al. [1] have reported similar negative $\mathrm{A}-\mathrm{V}$ differences in normal fasting subjects in early morning hours. Measurements in our control population have not demonstrated this. In any event, our patients demonstrated negative $\mathrm{A}-\mathrm{V}$ potassium differences postprandially as well as when fasting. Intravenous infusion of glucose did produce a positive $\mathrm{A}-\mathrm{V}$ difference in serum potassium in one patient. Oral glucose administration did not produce this in another patient. ZrerLer et al. [11] and Gros et al. [3] each reported negative A-V differences in serum potassium on recovery from paralysis in patients with hypokalemic periodic paralysis with or without potassium chloride administration. Gros et al. [3] also demonstrated negative $\mathrm{A}-\mathrm{V}$ differences with vigorous muscle contraction in normal subjects. Both potassium chloride administration and vigorous exercise were followed by positive $\mathrm{A}-\mathrm{V}$ potassium differences in our patients. We have observed previously, as well as in this study, equally large negative A-V serum potassium differences in patients with hyperkalemic periodic paralysis at the onset of attacks, with reversal on recovery [5]. In patients described in this report, worsening of myotonia following exercise or potassium chloride infusion, or development of flaccid paralysis were associated with positive A-V potassium differences. The differences became negative on recovery. We would expect that $\mathrm{A}-\mathrm{V}$ differences would be positive in these patients at night as demonstrated by ZIERLer et al. but, unfortunately, we were unable to obtain specimens at that time. The reproducibility of these findings makes sudden changes in blood flow an unlikely explanation for them.

We do not understand the significance of the negative A-V differences in $\mathrm{K}$ concentration in serum except as a marker. We believe, however, that the negative $\mathrm{A}-\mathrm{V}$ differences in the basal state separate these patients from those with hyperkalemic periodic paralysis. It remains to be seen whether this is true of all patients with paramyotonia or if our present patients form still another syndrome. Our belief is strengthened by failure to demonstrate spontaneous increases in $\mathrm{K}$ concentration in serum with onset of symptoms or with 
exercise followed by rest, although negative evidence must always be regarded with some skepticism.

The only known common factor in the patients with myotonia or paralytic syndromes frequently associated with myotonia, not shared by syndromes without myotonia, is an increase of muscle water between attacks [4]. During attacks of all the periodic paralyses and related conditions, there may be an increase in muscle water and sodium, although the evidence for this rests on rather meager data and observations of increased muscle size in attacks [4]. The significance of increased muscle water content is unknown. In some patients it suggests release of previously bound intracellular solute since extracellular osmolality has been normal when measured. There are no reports of muscle water and electrolyte contents in those rare patients reported to have myotonia with hypokalemic periodic paralysis, a syndrome ordinarily associated with decreased muscle water between attacks [4]. The data are too few for further speculation about this possible correlation.

\section{References and Notes}

1. Andres, R.; Gader, G.; Goldman, P. and ZierLER, K. L. : Net potassium movement between resting muscle and plasma in man in the basal state and during the night. J. clin. Invest. 36: 723 (1957).

2. Drager, G.A.; Hammill, J.F. and Shy, G.M.: Paramyotonia congenita. Amer.med. Ass. Arch. Neurol. Psychiat. 80: 1 (1958).

3. Grob, D.; Johns, R.J. and Liljestrand, A.: Potassium movement in patients with familial periodic paralysis: Relationship to defect in muscle function. Amer.J. Med. 23: 356 (1957).

4. Klein, R.: Periodic paralysis; in: L. I. Gardner: Endocrine and genetic diseases of childhood, p. 704 (Saunders, Philadelphia 1969).

5. Klein, R.; Egan, T. and Ustrer, P.: Changes in sodium, potassium and water in hyperkalemic periodic paralysis. Metabolism 9: 1005 (1960).

6. KleIN, R.; Haddow, J.E.; Kind, G. and CockBURN, F.: Effect of cold on muscle potentials and electrolytes. Metabolism 17: 1094 (1968).

7. Layzer, R.B.; Lovelace, R.E. and Rowland, L.P.: Hyperkalemic periodic paralysis. Arch. Neurol., Chicago 16: 455 (1967).
8. Poskanzer, D. G. and Kerr, D. N. S. : A third type of periodic paralysis with nomokalemia and favorable response to sodium chloride. Amer.J. Med. 31: 328 (1961).

9. Tompkins, V.; Lascelles, R.G. and MacKinney, B.: The relief of myotonia by use of potassium binding resin. J.Neurol. Neurosurg. Psychiat. 22: 50 (1959).

10. Van der Meulen, J.P.; Gilbert, G.J. and Kane, C. A.: Familial hyperkalemic paralysis with myotonia. New Engl.J. Med. 264: 1 (1961).

11. Zierler, K. L. and Andres, R.: Movement of potassium into skeletal muscle during spontaneous attacks in family periodic paralysis. J.clin. Invest. 36: 730 (1957).

12. Bio-Science Laboratory, Van Nuys, GA.

13. Informed consent was obtained for all subjects in this study.

14. Presented in part at XII International Congress of Pediatrics, Mexico City, December 6, 1968, and at the Annual Meeting of the American Pediatric Society, Atlantic City, New Jersey, May 3, 1969.

15. The authors are very grateful for the kindness of Dr. Davio Dawson, Peter Bent Brigham Hospital, Boston in performing the enzyme analyses in the excised muscle and to Dr. Robert MAaNeary for his light and electron-microscopic studies. We are indebted to Dr. Angelo Eraklis for his cooperation in performing the biopsy on patient $A$. Dr. E. Ouellette and Dr. R. Adams not only allowed us to examine patient $D$ but supplied us with a muscle sample from their biopsy. We are grateful to Dr. S.H. Greenblatt and Dr. N. Geschwind for allowing us to study patient $A$ and to report their findings, to Dr. T.Henzle for referring patient $C$ for studies, and to Dr. J. ConNelly for referring patients $B-1, B-2$, and $B-3$ to us. As usual, we are indebted to the nursing staff of the Pediatric Clinical Research Center for their assistance in making the observations, and to Mrs. P. Usher and Mrs. V.MAYO for performing all the laboratory analyses.

16. Supported by National Institutes of Health Grant no. FR-103-05.

17. Request for reprints should be addressed to RoBert Klein, M.D., Pediatric Glinical Center, Boston City Hospital, Boston, MA 02118 (USA). 18. Accepted for publication October 9, 1969. 